\title{
MAGNETOGRAM MEASURES OF TOTAL NONPOTENTIALITY FOR PREDICTION OF SOLAR CORONAL MASS EJECTIONS FROM ACTIVE REGIONS OF ANY DEGREE OF MAGNETIC COMPLEXITY
}

\author{
D. A. Falconer, ${ }^{1}$ R. L. Moore, and G. A. Gary \\ Marshall Space Flight Center, SD50, Space Science Department, Huntsville, AL 35812; \\ david.a.falconer@nasa.gov, ron.moore@nasa.gov, allen.gary@nasa.gov \\ Received 2008 March 3; accepted 2008 May 19
}

\begin{abstract}
For investigating the magnetic causes of coronal mass ejections (CMEs) and for forecasting the CME productivity of active regions, in previous work we have gauged the total nonpotentiality of a whole active region by either of two measures, $L_{\mathrm{SSM}}$ and $L_{\mathrm{SGM}}$, two measures of the magnetic field along the main neutral line in a vector magnetogram of the active region. This previous work was therefore restricted to nominally bipolar active regions, active regions that have a clearly identifiable main neutral line. In the present paper, we show that our work can be extended to include multipolar active regions of any degree of magnetic complexity by replacing $L_{\mathrm{SSM}}$ and $L_{\mathrm{SGM}}$ with their generalized counterparts, $\mathrm{WL}_{\mathrm{SS}}$ and $\mathrm{WL}_{\mathrm{SG}}$, which are corresponding integral measures covering all neutral lines in an active region instead of only the main neutral line. In addition, we show that for active regions within 30 heliocentric degrees of disk center, $\mathrm{WL}_{\mathrm{SG}}$ can be adequately measured from line-of-sight magnetograms instead of vector magnetograms. This approximate measure of active-region total nonpotentiality, ${ }^{L} \mathrm{WL}_{\mathrm{SG}}$, with the extensive set of 96 minute cadence full-disk line-of-sight magnetograms from $\mathrm{SOHO} \mathrm{MDI}$, can be used to study the evolution of active-region total nonpotentiality leading to the production of CMEs.
\end{abstract}

Subject headings: Sun: coronal mass ejections (CMEs) - Sun: magnetic fields

\section{INTRODUCTION}

Major coronal mass ejections (CMEs) are the greatest explosions in the solar system, magnetic plasmoids that explode from the Sun, expand to heliocentric angular widths of $90^{\circ}$ or more, plow through the solar wind, and produce severe space weather throughout much of the heliosphere (Suess \& Tsurutani 1998; Reames 2001). Each such great explosion is a release of $\sim 10^{32}$ ergs of magnetic energy: the plasmoid carries $\sim 10^{16} \mathrm{~g}$ of plasma and attains a super-Alfvénic speed of $\sim 10^{3} \mathrm{~km} \mathrm{~s}^{-1}$ (Wagner 1984; Kahler 1987). The resulting bow shock can accelerate protons and other ions to cosmic-ray energies of $100 \mathrm{MeV}$ or more, producing a far-reaching storm of solar energetic particles in the heliosphere (Krimigis 1992; Gosling 1996; Reames 1999). If such a CME intercepts the Earth, it bashes the Earth's magnetosphere, generating a storm of particle radiation within the magnetosphere.

The strongest CMEs erupt from magnetically strong regions of the Sun, active regions having sunspots, but many sunspot active regions produce no CMEs. Identification and quantification of active-region magnetic conditions that cause or prevent CME production is basic information both for understanding the magnetic explosion and for space weather forecasting.

It is observed that CMEs often erupt from active regions that have a filament and/or filament channel and from active regions that have a delta sunspot (Webb et al. 1976; Zirin \& Liggett 1987; Wang \& Zhang 2007). Filaments, filament channels, and delta sunspots mark magnetic fields that are greatly deformed (sheared and twisted) from their zero free energy potential-field configurations (Zirin 1988; Martin 1998). Hence, the presence of a filament, a filament channel, or a delta sunspot indicates that the active-region field has a large store of free energy, magnetic energy that in principle can be tapped to drive a CME explosion.

\footnotetext{
${ }^{1}$ Physics Department, University of Alabama at Huntsville, Huntsville, AL
}

The observed tendency for the source region of a CME to have a delta sunspot and/or a filament/filament channel has therefore engendered the standard view that an ample store of free magnetic energy in the source region is a necessary precondition for the production of a CME (Svestka 1976; Sturrock 1980; Svestka et al. 1992).

In coronal X-ray images, such as from Yohkoh or Hinode, strongly nonpotential active regions often have overall $S$-shaped or reverse- $S$-shaped sigmoid structure that vividly displays shear and twist in the magnetic field across the active region (Canfield et al. 1999). In a typical, nominally bipolar, nonpotential active region, the middle of the sigmoid traces the magnetic polarity inversion line (neutral line) through the active region (e.g., Moore et al. 2001). Canfield et al. (1999) found that whether an active region produces any CMEs during disk passage is significantly correlated with whether it has recognizable sigmoid structure in coronal X-ray images. For their sample of about a hundred active regions, $\sim 65 \%$ either appeared sigmoidal and produced CME eruptions or appeared nonsigmoidal and produced no CME eruptions. In addition, using sunspot area as an indicator of the magnetic size of an active region, Canfield et al. (1999) found that whether an active region is CME productive depends about as strongly on the active region's magnetic size as it does on the active region's apparent sigmoidality. For the nonsigmoidal active regions as well as for the sigmoidal active regions, $\sim 65 \%$ either had sunspot area greater than 50 millionths of the disk and were CME productive or had sunspot area less than 50 millionths of the disk and were not CME productive.

Motivated by the work of Canfield et al. (1999), in a series of papers we have adopted a number of whole-active-region magnetic quantities that can be directly measured from an activeregion magnetogram, and have assessed these as predictors of active-region CME productivity (Falconer 2001; Falconer et al. $2002,2003,2006)$. One of these magnetogram measures, $\Phi$, is the active region's total magnetic flux above a threshold strength 

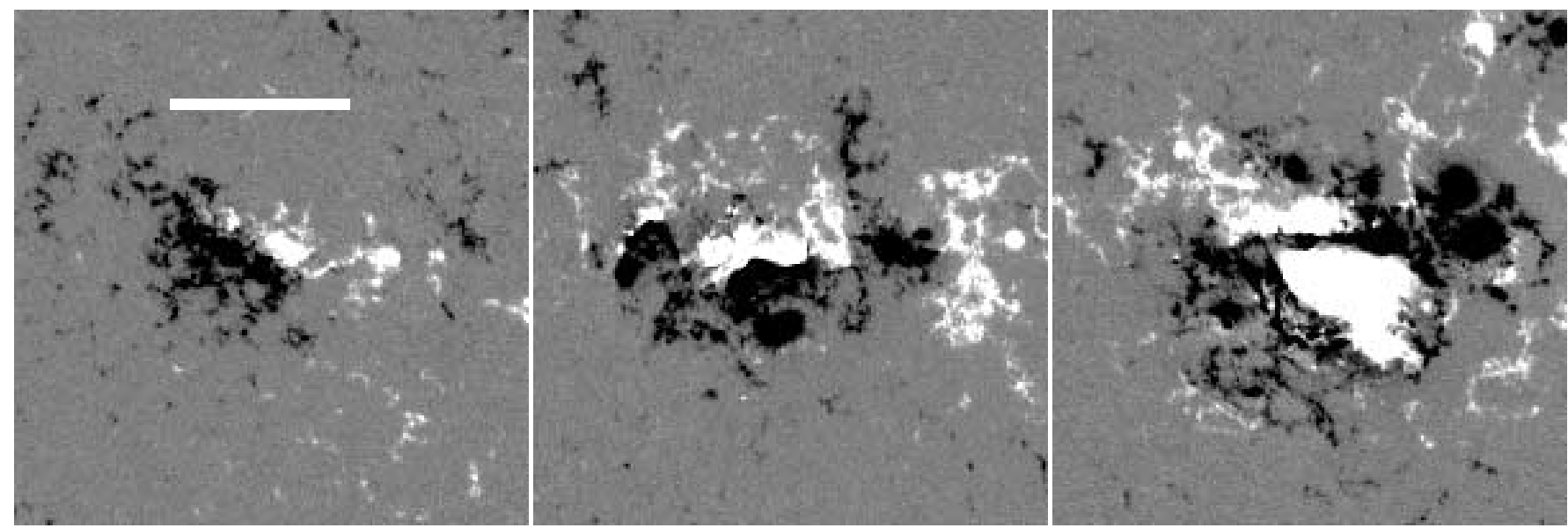

FIG. 1.-Display of the diverse magnetic complexity of active regions, ranging from purely bipolar (left) to predominantly bipolar (middle) to complex multipolar (right) arrangements of magnetic flux. Each panel is a gray-scale line-of-sight magnetogram of a different active region with positive-polarity flux shown light and negative-polarity flux dark. Each is from a full-disk MDI magnetogram taken when the active region was near disk center and has north up and west right, and all three have the same spatial and magnetic scales. The white bar in the left panel is $100,000 \mathrm{~km}$ long. Left: Example of a purely bipolar active region (AR 9370 at W20 $0^{\circ}$, N1 $0^{\circ}$ on 2001 March 9). It has a single flux domain of each polarity and a single neutral line between them. Middle: Example of a predominantly bipolar active region (AR 9077 at $\mathrm{W} 6^{\circ}, \mathrm{N} 17^{\circ}$ on 2000 July 14). It has an obvious main neutral line, the long meandering one between the dominant positive-polarity domain and the negative-polarity domain, plus a secondary neutral line, the short one between the negative domain and the separate positive domain at the west end of the active region. Right: Example of a complex multipolar active region ( $\mathrm{AR} 10486$ at $\mathrm{E} 10^{\circ}, \mathrm{S} 18^{\circ}$ on 2003 October 28). It does not have a clearly dominant main neutral line, but two major neutral lines and a scattering of lesser ones.

set by the noise level of the magnetogram. This is a more direct measure of the magnetic size of an active region than is sunspot area. The measure $\Phi$ is well defined for any active region, regardless of the degree of intermixing of positive-polarity flux and negative-polarity flux. Each of the other magnetogram measures that we have assessed for CME prediction is a measure of the overall nonpotentiality of an active region. Most active regions, including most sigmoidal active regions, are nominally bipolar, ones that have a dominant pair of opposite-polarity flux domains, the neutral line between them being the main neutral line crossing the active region (e.g., Moore 1992). In Figure 1, the active region in the left panel is purely bipolar, and so has only a single neutral line. The active region in the middle panel has in its leading end a secondary neutral line that is separate from the main neutral line, which is the one that meanders across most of the active region. Both of these active regions are nominally bipolar. In contrast, the active region in the right panel has a more complex mix of opposite-polarity magnetic flux, resulting in no neutral line being unquestionably the main neutral line. Such magnetically complex active regions are not nominally bipolar, and we refer to them as multipolar active regions. In nominally bipolar sigmoidal active regions, the middle of the sigmoid traces the main neutral line and, as is confirmed by vector magnetograms, this reveals that magnetic shear is concentrated along the main neutral line (e.g., Falconer et al. 1997; Moore et al. 2001). For these reasons, the measures of active-region nonpotentiality that we have studied in our previous papers are defined for and limited to nominally bipolar active regions. Each is a measure of the overall nonpotentiality of most of the active region's magnetic field, the field that arches over the main neutral line.

In our most extensive previous study (Falconer et al. 2006), we examined the CME-prediction performance of six wholeactive-region magnetogram measures: the magnetic flux content and five measures of nonpotentiality. These six quantities were extracted from each of 36 vector magnetograms of nominally bipolar active regions, each of which was within $\sim 30^{\circ}$ of disk center when the vector magnetogram was taken by the Marshall Space Flight Center (MSFC) vector magnetograph. The magnetic flux content $\Phi$ is measured from the vertical-field component of the vector magnetogram. Three of the nonpotentiality measures are measures of the total nonpotentiality of an active region. That is, each of these measures is a gauge of the active region's total free magnetic energy. These three measures are (1) $L_{\mathrm{SSM}}$, the length of strong-shear main neutral line; (2) $I_{N}$, the net electric current arching over the main neutral line; and (3) $L_{\mathrm{SGM}}$, the length of strong-gradient main neutral line. The quantities $L_{\mathrm{SSM}}$ and $I_{N}$ are obtained from the full deprojected vector magnetogram, that is, from a combination of the horizontal-field component and the vertical-field component. In contrast, $L_{\mathrm{SGM}}$ is measured from only the vertical-field component. Each of these three measures is proportional to the diameter of the active region. The other two nonpotentiality measures have no intrinsic dependence on the size of the active region. Each characterizes the overall twist in the active region's magnetic field in the manner of the $\alpha$ parameter in a force-free field; each is a gauge of the nonpotential energy per unit of magnetic flux in the active region. These two measures are (1) $\alpha_{\mathrm{IN}}$, the net-current alpha, which is proportional to the ratio of the net current $I_{N}$ to the flux content $\Phi$; and (2) $\alpha_{\mathrm{BC}}$, the best constant alpha, which is the $\alpha$ of the constant- $\alpha$ forcefree field that best fits the vector magnetogram. Each of these magnetic twist measures is obtained from a combination of the vertical-field component and the horizontal-field component of the deprojected vector magnetogram.

For the sample of nominally bipolar active regions measured in Falconer et al. (2006), each of the three measures of total nonpotentiality $\left(L_{\mathrm{SSM}}, I_{N}, L_{\mathrm{SGM}}\right)$ is strongly correlated with the other two, and each has a $\sim 75 \%$ success rate as a predictor of whether an active region will or will not produce a CME in the coming few days after the measured magnetogram. Thus, at least for CME prediction, $L_{\mathrm{SSM}}, I_{N}$, and $L_{\mathrm{SGM}}$ are nearly equivalent measures of active-region total nonpotentiality. In addition, the $\sim 75 \% \mathrm{CME}-$ prediction success rate, being higher than the $\sim 65 \%$ success rate found by Canfield et al. (1999) for active-region sigmoidality and for active-region sunspot area, indicates that the total nonpotentiality is a stronger determinant of an active region's CME productivity than is either the active region's sigmoidality or its 
magnetic size. Another result from Falconer et al. (2006) is that the two measures of overall magnetic twist $\left(\alpha_{\mathrm{IN}}, \alpha_{\mathrm{BC}}\right)$ are strongly correlated and each has a CME prediction success rate of $\sim 65 \%$. This indicates that (1) for CME prediction, $\alpha_{\mathrm{IN}}$ and $\alpha_{\mathrm{BC}}$ are nearly equivalent measures of the overall twist in an active region's magnetic field, and (2) the total nonpotentiality is a stronger determinant of an active region's CME productivity than is the overall degree of magnetic twist. The $\sim 65 \%$ CME-prediction success rate of the twist measures matches the $\sim 65 \%$ success rate found by Canfield et al. (1999) for active-region sigmoidality. This match is consistent with sigmoidality being, like the twist measures, a signature of an active region's overall nonpotentiality that has no intrinsic dependence on the size of the active region. A further result from the Falconer et al. (2006) sample of nominally bipolar active regions is that the flux content $\Phi$ has no correlation with either of the twist measures, but has about the same CME-prediction success rate as the twist measures, $\sim 65 \%$. This indicates that magnetic size and overall magnetic twist are independent but about equally strong determinants of an active region's CME productivity. The $\sim 65 \%$ CME-prediction success rate of $\Phi$ matches the $\sim 65 \%$ success rate found by Canfield et al. (1999) for active-region sunspot area, as is reasonable since $\Phi$ and sunspot area are each a measure of an active region's magnetic size. Finally, it is consistent that the total nonpotentiality measures $L_{\mathrm{SSM}}, I_{N}$, and $L_{\mathrm{SGM}}$ each have a higher CME-prediction success rate than do either the twist measures $\alpha_{\mathrm{IN}}$ and $\alpha_{\mathrm{BC}}$ or the flux content $\Phi$, because the total nonpotentiality of an active region is roughly the product of its overall magnetic twist and its magnetic size: $I_{N} \propto \alpha_{\mathrm{IN}} \Phi$.

The above results of Falconer et al. (2006) establish that the examined six whole-active-region magnetogram measures are useful for assessing basic magnetic causes of CMEs and for forecasting the occurrence of CMEs. However, except for the magnetic flux content $\Phi$, each of these measures, by design, is limited to nominally bipolar active regions: each is undefined for multipolar active regions. Because the most CME-productive active regions, especially those that have delta sunspots, typically are multipolar, we want to have similar magnetogram measures for multipolar active regions. Because we know that for nominally bipolar active regions magnetogram measures of total nonpotentiality are stronger predictors of an active region's CME productivity than are magnetogram measures of overall magnetic twist or magnetic size, we especially want to have magnetogram measures of total nonpotentiality for multipolar active regions. Fortunately, two of the total nonpotentiality measures for nominally bipolar active regions, $L_{\mathrm{SSM}}$ and $L_{\mathrm{SGM}}$, can be simply redefined to include all strong-field neutral lines in the active region instead of only the main neutral line. These two direct extensions of $L_{\mathrm{SSM}}$ and $L_{\mathrm{SGM}}$, which we denote by $L_{\mathrm{SSA}}$ and $L_{\mathrm{SGA}}$, can be measured from active-region magnetograms of any degree of complexity, from purely bipolar to multipolar.

In this paper, again from measurement of MSFC vector magnetograms of active regions, some nominally bipolar and some multipolar, we demonstrate four things. First, we show that for nominally bipolar active regions, as CME predictors, $L_{\mathrm{SSA}}$ and $L_{\mathrm{SGA}}$ work as well as $L_{\mathrm{SSM}}$ and $L_{\mathrm{SGM}}$. Second, we show that a further refinement of these two neutral-line measures of activeregion total nonpotentiality, namely weighted integral analogs of $L_{\mathrm{SSA}}$ and $L_{\mathrm{SGA}}$, denoted by $\mathrm{WL}_{\mathrm{SS}}$ and $\mathrm{WL}_{\mathrm{SG}}$, greatly reduce the uncertainty in the measurement for active regions of moderate to low nonpotentiality. Third, we show that as CME predictors, $\mathrm{WL}_{\mathrm{SS}}$ and $\mathrm{WL}_{\mathrm{SG}}$ measured from either nominally bipolar active regions or multipolar active regions work as well as $L_{\mathrm{SSM}}$ and $L_{\mathrm{SGM}}$ measured from nominally bipolar active regions. Finally, we show that for CME prediction $\mathrm{WL}_{\mathrm{SG}}$ can be adequately measured from the line-of-sight component of the vector magnetogram, instead of from the vertical-field component, when the active region is within $30^{\circ}$ of disk center. (For active regions within $30^{\circ}$ of disk center, the line-of-sight field component also gives a good approximation for the flux content $\Phi$; Falconer et al. 2006.) This opens a new window for our approach to finding and ranking measurable magnetic preconditions for CME production: we can tap the archive of full-disk line-of-sight magnetograms from the Solar and Heliospheric Observatory (SOHO) MDI, which has over $10 \mathrm{yr}$ of nearly continuous coverage at a 96 minute cadence, to track the evolution of active-region total nonpotentiality and magnetic flux content $\left(\mathrm{WL}_{\mathrm{SG}}\right.$ and $\left.\Phi\right)$ in active regions within $30^{\circ}$ of disk center and investigate how this evolution relates to active-region $\mathrm{CME}$ productivity.

\section{DATA AND MEASUREMENTS}

\subsection{Sample of Active-Region Vector Magnetograms}

The results reported in this paper are from MSFC vector magnetograms of active regions. We measured a total of 56 vector magnetograms, each of a different active region. For each active region, the measurements were made after deprojection of the observed vector magnetogram to disk center. That is, we first transformed the observed line-of-sight field and transverse-field components of the vector magnetogram to obtain the vertical and horizontal components of the observed vector field. Before this transformation could be done, the $180^{\circ}$ ambiguity in the direction of the transverse field in the observed vector magnetogram had to be removed. We used the methods described in Falconer et al. (2006) to directionalize the observed vector magnetogram and to transform the directionalized magnetogram to its vertical-field and transverse-field components.

To be included in our sample, each active region had to meet two conditions at the time of its magnetogram. One condition was that the active region had be no more than about $30^{\circ}$ in longitude from central meridian. This condition limits deprojection errors in the measured magnetogram. For the 56 active regions in our sample, none had a heliocentric angle from disk center greater than $38^{\circ}$, and for 41 of these active regions this angle was no more than $30^{\circ}$.

The other condition for inclusion in our sample was that the active region be a "strong-field" active region relative to the sensitivity of the MSFC vector magnetograms. Three of our measures of an active region's total nonpotentiality $\left(L_{\mathrm{SSM}}, L_{\mathrm{SSA}}, \mathrm{WL}_{\mathrm{SS}}\right)$ are measured from the observed horizontal field on neutral lines of the active region. In order for the measured value of $L_{\mathrm{SSM}}$, $L_{\mathrm{SSA}}$, or $\mathrm{WL}_{\mathrm{SS}}$ to be representative of the active region's total nonpotentiality, the strength of the observed horizontal field must be well above the noise level of the magnetogram over enough of the length of the neutral lines: the total length of such strongfield neutral-line intervals in the active region needs to be comparable to the overall magnetic span of the active region. The $1 \sigma$ noise levels of the MSFC vector magnetograms are $50 \mathrm{G}$ for the line-of-sight component and $75 \mathrm{G}$ for the transverse component (Gary et al. 1987). We restrict our measurements of all of our measures of active-region total nonpotentiality $\left(L_{\mathrm{SSM}}, L_{\mathrm{SGM}}, L_{\mathrm{SSA}}\right.$, $L_{\mathrm{SGA}}, \mathrm{WL}_{\mathrm{SS}}, \mathrm{WL}_{\mathrm{SG}}$ ) to those intervals of an active region's neutral lines on which the field is strong enough to be measured well by the MSFC vector magnetograph. For our neutral-line gradient measures $\left(L_{\mathrm{SGM}}, L_{\mathrm{SGA}}, \mathrm{WL}_{\mathrm{SG}}\right)$, we take the strong-field intervals of neutral line to be those on which the potential horizontal field computed from the vertical-field component of the magnetogram is greater than $150 \mathrm{G}$. We define the length $L_{S}$ to be the total length 
of these strong-field intervals of neutral line in the active region. We define the magnetic area of an active region to be the area of the active region in which the vertical-field component of the vector magnetogram is greater than $100 \mathrm{G}$. The square root of this area is a characteristic length $L_{\mathrm{AR}}$ that is representative of the overall magnetic span of the active region. For an active region to be a strong-field active region that could be included in our sample, we required that $L_{S}$ be greater than $0.75 L_{\mathrm{AR}}$.

The arrangement of the positive-polarity and negative-polarity domains in the line-of-sight component of an active region's vector magnetogram largely defines the degree of complexity of the field configuration. From inspection of the magnetograms, we sorted our 56 active regions into three classes of overall polarity arrangement: (1) purely bipolar (those having only one neutral line); (2) predominantly bipolar (those having an obvious main neutral line); and (3) multipolar (those having no obvious main neutral line, but two or more comparably long separate neutral lines). An example of one of our active regions of each class is shown in Figure 1, where each active region is viewed in an MDI magnetogram when the active region was near disk center. In our previous papers, we have restricted our sample to active regions that were either purely bipolar or predominantly bipolar, because our previous neutral-line measures of active-region total nonpotentiality ( $L_{\mathrm{SSM}}$ and $L_{\mathrm{SGM}}$ ) were defined only for active regions that had a clear-cut main neutral line. In the present paper, we generalize these measures to include the strong-field intervals of all of the neutral lines of an active region regardless of the complexity of its polarity arrangement. To determine whether the generalized measures perform as well as the main-neutral-line measures for CME prediction, we examine the CME-prediction performance of the generalized measures for two complementary subsets of our sample and for the entire sample of 56 active regions. The two subsets are (1) the subset of 40 active regions that are either purely bipolar or predominantly bipolar, which we call the bipolar sample, and (2) the subset of 16 multipolar active regions, which we call the multipolar sample. We call the full sample of 56 active regions the combined sample.

For a separate purpose, we use another subset of the combined sample. This is the subset of 41 active regions each of which was within $30^{\circ}$ of disk center when its vector magnetogram was taken. We use this sample to show that, for forecasting CMEs from active regions this close to disk center, a proxy measure of active-region total nonpotentiality $\left({ }^{L} \mathrm{WL}_{\mathrm{SG}}\right)$ that can be obtained from a line-ofsight magnetogram performs as well as our corresponding measures $\left(\mathrm{WL}_{\mathrm{SG}}\right.$ and $\left.\mathrm{WL}_{\mathrm{SS}}\right)$ that require a vector magnetogram.

Of the 56 active regions in our combined sample, 47 occurred during the period from the beginning of $\mathrm{SOHO}$ (1996) to the end of Yohkoh (2001). These 47 active regions are all of those active regions observed by the MSFC vector magnetograph in this period that have a vector magnetogram that satisfies our two selection criteria. The other nine are a smattering of active regions that occurred either earlier during Yohkoh (1991-1996) or after 2001. Hence, with regard to the distribution of the degree of nonpotentiality among active regions, our combined sample is a nearly unbiased random sample of the population of strong-field active regions produced by the Sun.

\subsection{Magnetogram Measures of Active-Region Total Nonpotentiality}

The six measures of active-region total nonpotentiality that we measured in our deprojected vector magnetograms are defined as follows. The length of strong-shear main neutral line, $L_{\mathrm{SSM}}$, is the total length of all main-neutral-line intervals on which (1) the observed horizontal field is greater than $150 \mathrm{G}$, and (2) the shear

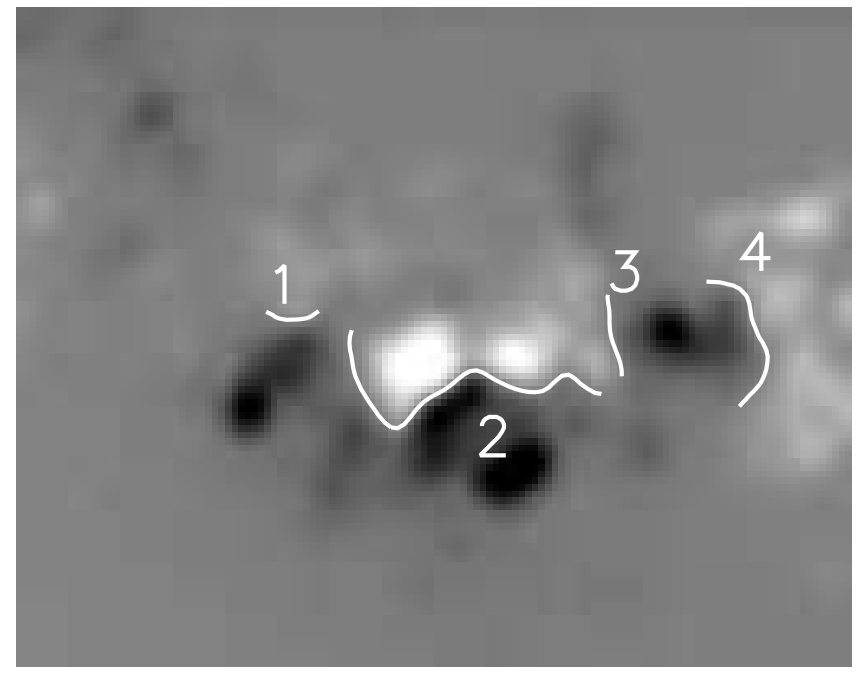

FIG. 2.-Strong-potential-field intervals of the neutral lines in the predominantly bipolar active region from the middle panel of Fig. 1. This gray-scale magnetogram is the vertical-field component of the deprojected MSFC vector magnetogram of that active region. Each white curve traces a neutral-line interval throughout which the potential horizontal field computed from this vertical-field magnetogram is greater that $150 \mathrm{G}$. Intervals 1,2 , and 3 are the strong-potentialfield intervals of the main neutral line of this active region, and interval 4 is the strong-potential-field interval of the secondary neutral line. The neutral-line magnetic-gradient measures of an active region's total nonpotentiality used in this paper are restricted to such strong-potential-field intervals of the neutral lines. The neutral-line magnetic-shear measures of total nonpotentiality are restricted to analogous strong-observed-field intervals of the neutral lines, the intervals throughout which the horizontal component of the observed field is greater than $150 \mathrm{G}$.

angle, the angle between the observed horizontal field and the computed potential horizontal field, is greater than $45^{\circ}$. The length of strong-gradient main neutral line, $L_{\mathrm{SGM}}$, is the total length of all main-neutral-line intervals on which (1) the potential horizontal field is greater than $150 \mathrm{G}$, and (2) the horizontal gradient of the vertical component of the magnetic field is greater than $50 \mathrm{G} \mathrm{Mm}^{-1}$. The strong-potential-field intervals of neutral line in our example predominantly bipolar active region are shown in Figure 2. The measures $L_{\mathrm{SSM}}$ and $L_{\mathrm{SGM}}$ are defined only for active regions that have an obvious main neutral line. That is, they are defined for purely bipolar or predominantly bipolar active regions, but not for multipolar active regions. The other four of the six measures of active-region total nonpotentiality are generalized analogs of $L_{\mathrm{SSM}}$ and $L_{\mathrm{SGM}}$ that are defined for active regions of any degree of magnetic complexity. The length of strong-shear neutral line, $L_{\mathrm{SSA}}$, is the total length of all of the active region's neutral-line intervals on which (1) the observed horizontal field is greater than $150 \mathrm{G}$, and (2) the shear angle is greater than $45^{\circ}$. The length of strong-gradient neutral line, $L_{\mathrm{SGA}}$, is the total length of all neutral-line intervals on which (1) the potential horizontal field is greater than $150 \mathrm{G}$, and (2) the horizontal gradient of the vertical component of the magnetic field is greater than $50 \mathrm{G} \mathrm{Mm}^{-1}$. The shear-weighted integral length of neutral line, $\mathrm{WL}_{\mathrm{SS}}$, is the line integral of the shear angle (in radians) over all neutral-line intervals on which the observed horizontal field is greater than $150 \mathrm{G}$ $\left(\mathrm{WL}_{\mathrm{SS}}=\int \theta d l\right.$ where $\theta$ is the shear angle and $\mathrm{WL}_{\mathrm{SS}}$ has units of radians times kilometers). The shear-weighted integral length of neutral line, $\mathrm{WL}_{\mathrm{SS}}$, is the line integral of the shear angle (in radians) over all neutral-line intervals on which the observed horizontal field is greater than $150 \mathrm{G}\left(\mathrm{WL}_{\mathrm{SS}}=\int \theta d l\right.$ where $\theta$ is the shear angle and $\mathrm{WL}_{\mathrm{SS}}$ has units of radians times kilometers). The gradient-weighted integral length of neutral line, $\mathrm{WL}_{\mathrm{SG}}$, is the line integral of the vertical-field horizontal gradient over all 
TABLE 1

CME-Predication Success Rates for Our Various Measures, Samples, and Forecast Windows

\begin{tabular}{|c|c|c|c|c|c|c|}
\hline \multirow[b]{2}{*}{ SAMPLE } & \multirow[b]{2}{*}{ Measure } & \multicolumn{5}{|c|}{ FoRECAST WindOW } \\
\hline & & $\begin{array}{c}0-24 \mathrm{hr} \\
(\%)\end{array}$ & $\begin{array}{c}0-48 \mathrm{hr} \\
(\%)\end{array}$ & $\begin{array}{c}0-72 \mathrm{hr} \\
(\%)\end{array}$ & $\begin{array}{c}0-96 \mathrm{hr} \\
(\%)\end{array}$ & $\begin{array}{c}0-120 \mathrm{hr} \\
(\%)\end{array}$ \\
\hline \multirow[t]{6}{*}{ 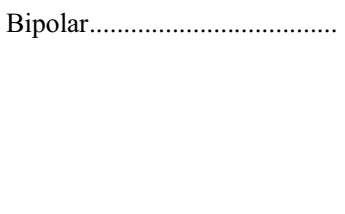 } & $L_{\mathrm{SSM}}$ & $80 \pm 6$ & $80 \pm 6$ & $85 \pm 6$ & $80 \pm 6$ & $65 \pm 8$ \\
\hline & $L_{\mathrm{SGM}}$ & $75 \pm 7$ & $80 \pm 6$ & $85 \pm 6$ & $70 \pm 7$ & $50 \pm 8$ \\
\hline & $L_{\mathrm{SSA}}$ & $80 \pm 6$ & $80 \pm 6$ & $80 \pm 6$ & $75 \pm 7$ & $65 \pm 8$ \\
\hline & $L_{\mathrm{SGA}}$ & $75 \pm 7$ & $75 \pm 7$ & $80 \pm 6$ & $75 \pm 7$ & $65 \pm 8$ \\
\hline & $\mathrm{WL}_{\mathrm{SS}}$ & $85 \pm 6$ & $80 \pm 6$ & $80 \pm 6$ & $70 \pm 7$ & $65 \pm 8$ \\
\hline & $\mathrm{WL}_{\mathrm{SG}}$ & $80 \pm 6$ & $70 \pm 7$ & $75 \pm 7$ & $75 \pm 7$ & $60 \pm 8$ \\
\hline \multirow[t]{2}{*}{ 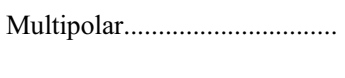 } & $\mathrm{WL}_{\mathrm{SS}}$ & $75 \pm 11$ & $88 \pm 8$ & $75 \pm 11$ & $75 \pm 11$ & $75 \pm 11$ \\
\hline & $\mathrm{WL}_{\mathrm{SG}}$ & $63 \pm 12$ & $75 \pm 11$ & $75 \pm 11$ & $63 \pm 12$ & $63 \pm 12$ \\
\hline \multirow[t]{2}{*}{ 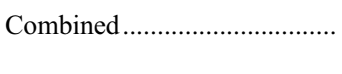 } & $\mathrm{WL}_{\mathrm{SS}}$ & $82 \pm 5$ & $82 \pm 5$ & $79 \pm 5$ & $68 \pm 6$ & $71 \pm 6$ \\
\hline & $\mathrm{WL}_{\mathrm{SG}}$ & $75 \pm 6$ & $71 \pm 6$ & $79 \pm 5$ & $71 \pm 6$ & $61 \pm 7$ \\
\hline \multirow[t]{2}{*}{ Combined, $<30^{\circ}$...................... } & $\mathrm{WL}_{\mathrm{SG}}$ & $80 \pm 6$ & $73 \pm 7$ & $76 \pm 7$ & $73 \pm 7$ & $61 \pm 8$ \\
\hline & ${ }^{L} \mathrm{WL}_{\mathrm{SG}}$ & $80 \pm 6$ & $83 \pm 6$ & $85 \pm 6$ & $73 \pm 7$ & $66 \pm 7$ \\
\hline
\end{tabular}

neutral-line intervals on which the potential horizontal field is greater than $150 \mathrm{G}\left[\mathrm{WL}_{\mathrm{SG}}=\int\left(\nabla B_{z}\right) d l\right.$, where $\nabla B_{z}$ is the horizontal gradient of the vertical magnetic field and $\mathrm{WL}_{\mathrm{SG}}$ has units of $\mathrm{G}]$.

For each of the 41 active regions that were strictly within $30^{\circ}$ of disk center when their vector magnetograms were taken, in addition to measuring the total nonpotentiality measure $\mathrm{WL}_{\mathrm{SG}}$ from the vertical-field component of the deprojected vector magnetogram, we also measured the corresponding proxy measure from the line-of-sight component of the non-deprojected original vector magnetogram. This proxy measure, ${ }^{L} \mathrm{WL}_{\mathrm{SG}}$, is the gradient-weighted integral length of neutral line in the line-of-sight magnetogram: the line integral of the transverse gradient of the line-of-sight field over all neutral-line intervals on which the potential transverse field (computed from the line-of-sight magnetogram) is greater than $150 \mathrm{G}$.

Using a Monte Carlo technique, for each measured value of each of the above seven measures of active-region total nonpotentiality, we computed the $1 \sigma$ uncertainty from the uncertainties in the line-of-sight and transverse components of the MSFC vector magnetogram. This procedure was the same as in our previous similar work, and is described in Falconer et al. (2006).

\subsection{Active-Region CME Productivity}

Our basic purpose in this paper is to determine how well each of our total nonpotentiality measures predicts an active region's forthcoming CME productivity, in the sense of predicting whether the active region will or will not produce a CME in the next few days after the time of the measured magnetogram. We do this by determining for our sample of active regions each measure's rate of correctly predicting this outcome for each of five progressively larger forward time windows: $0-24 \mathrm{hr}, 0-48 \mathrm{hr}, 0-72 \mathrm{hr}, 0-96 \mathrm{hr}$, and $0-120 \mathrm{hr}$. For this purpose, we determined for each window and each active region whether the active region was CME productive (produced one or more CMEs in the window) or was not CME productive (produced no CMEs in the window). We determined this for each active region by determining whether or not the active region produced any ejective flares in the time window. For most of our active regions, those that occurred during the Yohkoh mission (1991-2001), we determined this primarily from Yohkoh SXT coronal X-ray movies in combination with the progression of the Sun's soft X-ray flux observed by the Geostationary Operational Environmental Satellite (GOES). See Falconer et al. (2002) for details of the procedure. For these active regions, for those ejective flares with coverage by $\mathrm{SOHO}$, we used the SOHO EIT coronal EUV movies and the SOHO LASCO coronagraph movies, both from the $S O H O$ LASCO CME Catalog (Yashiro et al. 2004), to confirm that the ejective flare eruption produced a CME. For the few active regions that occurred after Yohkoh, the CME-production history of each active region was determined in like manner from these movies from the CME Catalog in combination with the GOES observations of the Sun's soft X-ray flux output. The correlation plots in this paper are all for the $0-72 \mathrm{hr}$ window. For each of our seven measures of total nonpotentiality, the CME-prediction success rates found from each of our various subsamples and for each of the five time widows are given in Table 1.

\subsection{Thresholds for Prediction of Active-Region CME Productivity}

To assess the strength of each of our total nonpotentiality measures as a predictor of active-region CME productivity, for each measure we adopt a threshold value below which an active region is expected to not be CME productive, and at and above which an active region is expected to be CME productive. In the manner of our approach in Falconer et al. (2006), we use the observed CME-production performance of the active regions in our samples to set the threshold values. For any given sample of $n$ active regions, for a given time window it will be found that some number $N$ of these active regions were CME productive and that the rest of them $(n-N)$ were not. In the present paper, for each given time window and sample of active regions, the CME-prediction threshold that we adopt for each total nonpotentiality measure is the $N$ th largest of the $n$ values measured for the set of $n$ active regions. For example, if during the given window 12 of the $n$ active regions were CME-productive, then for any given measure the 12 active regions that were the most nonpotential by this measure are expected to be CME productive and the rest $(n-12)$ are expected to not be CME productive. The CMEprediction success rate of a given measure, for a given window and a given sample, is the percentage of the $n$ active regions that were below threshold and produced no CMEs or were at or above threshold and were CME productive. That is, if $N$ of the $n$ active regions behaved as predicted by the threshold, then the CMEprediction success rate of the measure would be $(N / n) \times 100 \%$.

We also examine the agreement success rate for the agreement between a given pair of our measures with respect to their CMEprediction thresholds. The agreement success rate, for a given 
pair of measures, for a given sample of active regions and a given time window for CME production, is the percentage of active regions for which the measured values of the two measures are either both below threshold or both at or above threshold. That is, if $N$ of the $n$ active regions agreed in this way, then the agreement success rate, the rate of agreement of the two measures, would be $(N / n) \times 100 \%$

The statistical uncertainty in a CME-prediction success rate or in an agreement success rate can be estimated from the binomial theorem. This estimate is determined by both the sample size $n$ and the success rate $\pi$. From Lapin (1978), the estimated uncertainty $\sigma$ in the success rate is given by $\sigma=[\pi(1-\pi) n]^{1 / 2}$.

\section{RESULTS}

\subsection{Performance of the Main-Neutral-Line Measures}

To establish a base line of performance against which to gauge the performance of our generalized all-neutral-line measures of active-region total nonpotentiality, we first present the CMEprediction performance and the agreement performance of our two main-neutral-line measures of active-region total nonpotentiality, $L_{\mathrm{SSM}}$ and $L_{\mathrm{SGM}}$. For these two measures, the performance results reported in the present paper are from the vector magnetograms of purely or predominantly bipolar active regions comprising the bipolar sample defined in $\S 2$.1. In Falconer et al. (2006), employing essential the same procedure used here, we examined the performance of these two measures for a marginally smaller sample of purely or predominantly bipolar active regions. Of the 40 vector magnetograms in our present bipolar sample, 31 are from the set of vector magnetograms measured in Falconer et al. (2006). Consequently, the performance of $L_{\mathrm{SSM}}$ and $L_{\mathrm{SGM}}$ from our present bipolar sample is at most only marginally different from the performance found in Falconer et al. (2006).

For our bipolar sample, the 40 pairs of measured values of $L_{\mathrm{SSM}}$ and $L_{\mathrm{SGM}}$ and the uncertainties in these values are plotted logarithmically in Figure 3, where, as in all such correlation plots in this paper, the threshold lines and the dichotomy of CMEproductive active regions (black crosses) and non-CME-productive active regions (gray crosses) are for the $0-72 \mathrm{hr}$ window for CME production. For this sample and window, $L_{\mathrm{SSM}}$ and $L_{\mathrm{SGM}}$ happen to have the same CME-prediction success rate, $85 \% \pm 6 \%$. To within their uncertainties, this CME-prediction success rate is practically the same as those found for the 3 day ( $0-2$ days) window in Falconer et al. (2006): $72 \% \pm 6 \%$ for $L_{\mathrm{SSM}}$ and $88 \% \pm 6 \%$ for $L_{\mathrm{SGM}}$.

As can be seen in Figure 3, the fractional uncertainty in the measured value of $L_{\mathrm{SSM}}$ or $L_{\mathrm{SGM}}$ depends strongly on the magnitude of the measured value. The largest measured values, those from among the active regions having the largest total nonpotentiality, have among the smallest fractional uncertainties, and, on average, the uncertainties are progressively larger for progressively smaller values of $L_{\mathrm{SSM}}$ or $L_{\mathrm{SGM}}$, from active regions of progressively smaller total nonpotentiality. In particular, the average fractional uncertainty in the measured value of $L_{\mathrm{SSM}}$ or $L_{\mathrm{SGM}}$ is much larger for the active regions below threshold than for those above threshold. This trend in the uncertainties results in the correlation between $L_{\mathrm{SSM}}$ and $L_{\mathrm{SGM}}$ being much weaker in the below-threshold quadrant than in the above-threshold quadrant. However, even though the large uncertainties in the measured values results in the large scatter seen in the below-threshold points in Figure 3, for this sample, the agreement success rate of $L_{\mathrm{SSM}}$ and $L_{\mathrm{SGM}}$ relative to their thresholds is remarkably high, $90 \% \pm 5 \%$. This agreement success rate might be fortuitously high: for the set of 36 deprojected vector magnetograms measured in Falconer et al.

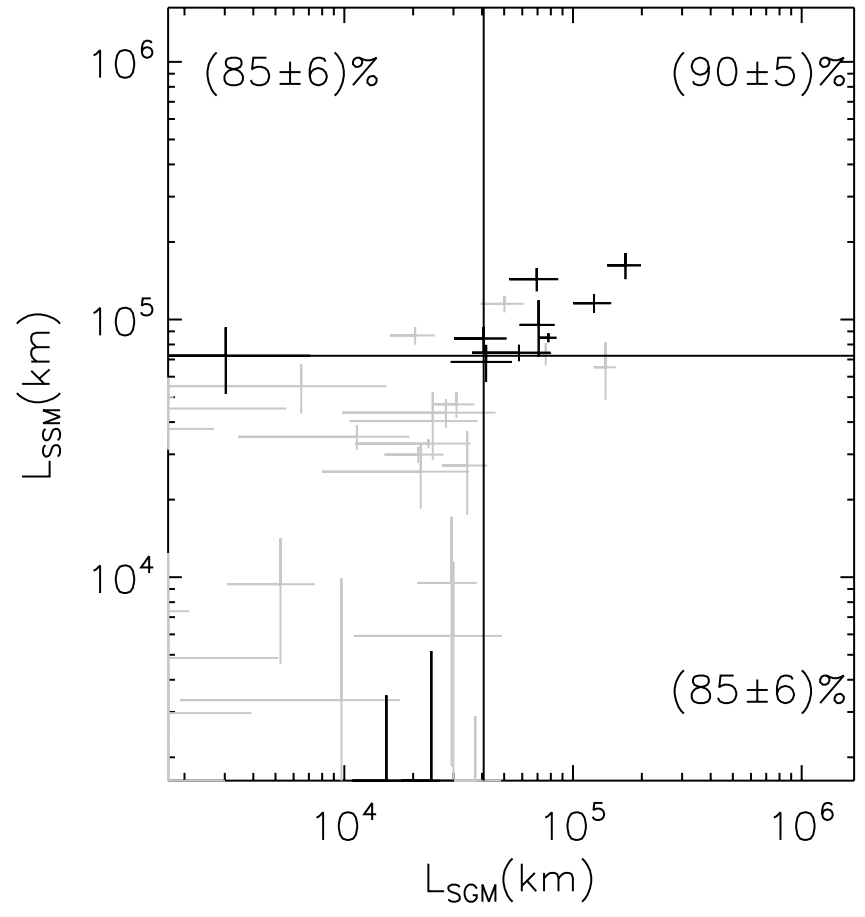

FIG. 3.-Correlation plot of the two nonweighted main-neutral-line measures of total nonpotentiality, $L_{\mathrm{SGM}}$ and $L_{\mathrm{SSM}}$, for our sample of 40 nominally bipolar active regions. The $\left(L_{\mathrm{SGM}}, L_{\mathrm{SSM}}\right)$ pair of values measured for each active region is plotted as a cross. The spans of the crosses give the $1 \sigma$ uncertainties in the measurements, and show that the uncertainties are much larger for weakly nonpotential active regions than for strongly nonpotential ones. The vertical line marks the CME expectation threshold for $L_{\mathrm{SGM}}$, for CME production by an active region in the next $72 \mathrm{hr}$ after its measured magnetogram; the horizontal line marks the corresponding threshold for $L_{\mathrm{SSM}}$. The darker crosses mark active regions that produced a CME in the $72 \mathrm{hr}$ window; the lighter crosses mark those that did not. The percentage near each axis is the CME-prediction success rate for that measure. The percentage in the upper right corner is the agreement success rate of the pairs of measured values, the percent of the active regions that have the two values either both below threshold or both at or above threshold. The uncertainty given for each percentage is the $1 \sigma$ statistical uncertainty estimated from the sample size and the success rate. This plot demonstrates our previous finding (Falconer et al. 2003, 2006): for nominally bipolar active regions within about $30^{\circ}$ of disk center $L_{\mathrm{SGM}}$ works as well as $L_{\mathrm{SSM}}$ for CME prediction.

(2006), the agreement success rate between $L_{\mathrm{SSM}}$ and $L_{\mathrm{SGM}}$ was $77 \% \pm 6 \%$.

\subsection{Performance of the All-Neutral-Line Measures for the Bipolar Sample}

We now turn to the performance of our generalized measures of active-region total nonpotentiality: the all-neutral-line measures $L_{\mathrm{SSA}}$ and $L_{\mathrm{SGA}}$, which are direct counterparts of $L_{\mathrm{SSM}}$ and $L_{\mathrm{SGM}}$; and the weighted all-neutral-line measures $\mathrm{WL}_{\mathrm{SS}}$ and $\mathrm{WL}_{\mathrm{SG}}$, which are refined counterparts of $L_{\mathrm{SSA}}$ and $L_{\mathrm{SGA}}$. The left panel of Figure 4, the correlation plot of $L_{\mathrm{SSA}}$ and $L_{\mathrm{SGA}}$ for the bipolar sample and the $0-72 \mathrm{hr}$ window, corresponds to the correlation plot of $L_{\mathrm{SSM}}$ and $L_{\mathrm{SGM}}$ in Figure 3. To within their uncertainties, the CME-prediction success rate for $L_{\mathrm{SSA}}(80 \% \pm 6 \%)$ is the same as that for $L_{\mathrm{SSM}}(85 \% \pm 6 \%)$, the CME-prediction success rate for $L_{\mathrm{SGA}}(80 \% \pm 6 \%)$ is the same as that for $L_{\mathrm{SGM}}(85 \% \pm 6 \%)$, and the agreement success rate between $L_{\mathrm{SSA}}$ and $L_{\mathrm{SGA}}(85 \% \pm 6 \%)$ is the same as that between $L_{\mathrm{SSM}}$ and $L_{\mathrm{SGM}}(90 \% \pm 5 \%)$.

In Figure 4 , the increase in the fractional uncertainty in $L_{\mathrm{SSA}}$ and $L_{\mathrm{SGA}}$ with decreasing magnitude of the measured value is comparable to that seen in Figure 3 for $L_{\mathrm{SSM}}$ and $L_{\mathrm{SGM}}$, again resulting in little correlation between below-threshold measured values of $L_{\mathrm{SSA}}$ and $L_{\mathrm{SGA}}$. In contrast, the right panel of Figure 4 , 

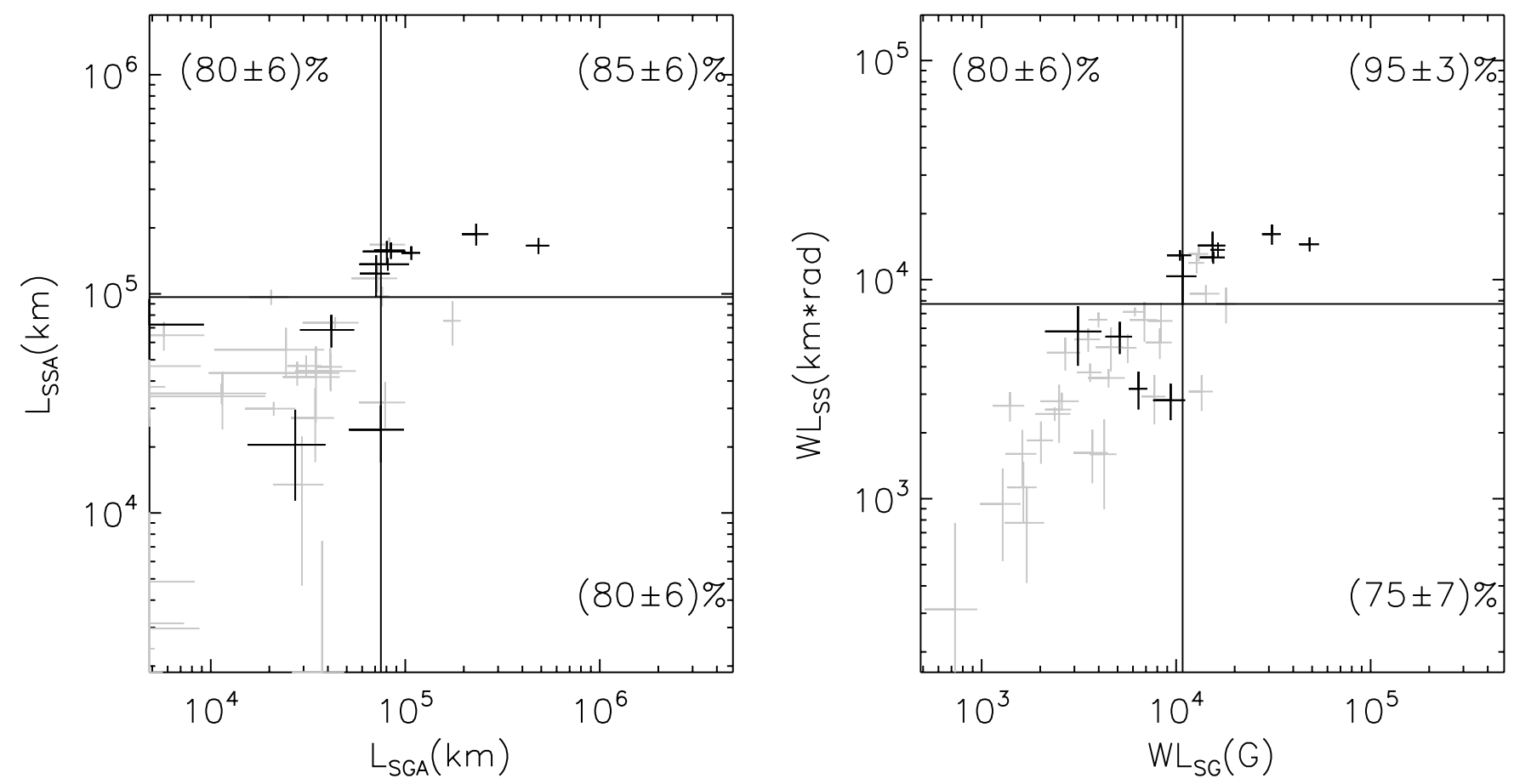

FIG. 4.-Correlation plots showing that for nominally bipolar active regions (1) the all-neutral-line generalized measures of total nonpotentiality perform as well as the main-neutral-line measures for prediction of CME production, and (2) for weakly nonpotential active regions, the measurement uncertainty in the weighted allneutral-line generalized measures is substantially less than for the nonweighted neutral-line measures. Left: Correlation plot of the two nonweighted all-neutral-line measures of total nonpotentiality, $L_{\mathrm{SGA}}$ and $L_{\mathrm{SSA}}$. Right: Correlation plot of the two weighted all-neutral-line measures of total nonpotentiality, WL $\mathrm{SG}_{\mathrm{SG}}$ and $\mathrm{WL}_{\mathrm{SS}}$. These plots are direct counterparts of the plot for the nonweighted main-neutral-line measures, $L_{\mathrm{SGM}}$ and $L_{\mathrm{SSM}}$, in Fig. 3, for the same sample of 40 bipolar active regions and for the $0-72 \mathrm{hr}$ forecast window. To within the statistical uncertainty, the CME-prediction success rate found here for each of these measures equals that found for the corresponding measure in Fig. 3.

the corresponding correlation plot for the weighted all-neutralline measures $\mathrm{WL}_{\mathrm{SS}}$ and $\mathrm{WL}_{\mathrm{SG}}$, shows a noticeably weaker increase of the uncertainty in a measured value with decreasing magnitude of the measured value. This results in $\mathrm{WL}_{\mathrm{SS}}$ and $\mathrm{WL}_{\mathrm{SG}}$ being obviously correlated throughout the observed range of active-region total nonpotentiality, above and below the threshold values. Comparison of the plots in Figures 3 and 4 shows that, for the bipolar sample, in addition to giving a significant reduction in the uncertainties of the measured values for active regions of average to small total nonpotentiality, $\mathrm{WL}_{\mathrm{SS}}$ and $\mathrm{WL}_{\mathrm{SG}}$ have CME-prediction success rates that are not only equal to each other within their uncertainties, but are also equal to any of those of the four less-refined measures. Figure 4 also shows that, for the bipolar sample, the agreement success rate between $\mathrm{WL}_{S \mathrm{SS}}$ and $\mathrm{WL}_{\mathrm{SG}}(95 \% \pm 3 \%)$ is marginally better than that between $L_{\mathrm{SSA}}$ and $L_{\mathrm{SGA}}(85 \% \pm 6 \%)$. Because $\mathrm{WL}_{\mathrm{SS}}$ and $\mathrm{WL}_{\mathrm{SG}}$ measure the total nonpotentiality of nominally bipolar active regions of moderate to small total nonpotentiality with much less fractional uncertainty than do $L_{\mathrm{SSA}}$ and $L_{\mathrm{SGA}}$, and have CMEprediction success rates equal to those of $L_{\mathrm{SSA}}$ and $L_{\mathrm{SGA}}$, it appears that $\mathrm{WL}_{S S}$ and $\mathrm{WL}_{\mathrm{SG}}$ are the better measures, and hence that these are the all-neutral-line measures of active-region total nonpotentiality that we should use from now on in our research on forecasting active-region CME productivity.

\subsection{Performance of the Weighted All-Neutral-Line Measures for the Multipolar and Combined Samples}

We have devised our weighted all-neutral-line measures of active-region total nonpotentiality, $\mathrm{WL}_{\mathrm{SS}}$ and $\mathrm{WL}_{\mathrm{SG}}$, because we want our investigations of the magnetic causes of CMEs and the predictability of CMEs from active-region magnetograms to cover active regions of all degrees of magnetic complexity. That is, we want our analyses to include the magnetically more complex, and more highly CME productive, multipolar active regions, and to no longer be limited to the relatively less magnetically complex purely and predominantly bipolar active regions. Whether $\mathrm{WL}_{\mathrm{SS}}$ and $\mathrm{WL}_{\mathrm{SG}}$ adequately serve this purpose hinges on whether these measures are adequately good predictors of the CME productivity of multipolar active regions, now that we have established (Fig. 4) that $\mathrm{WL}_{\mathrm{SS}}$ and $\mathrm{WL}_{\mathrm{SG}}$ are adequately good predictors of the CME productivity of purely and predominantly bipolar active regions.

The performances of $\mathrm{WL}_{\mathrm{SS}}$ and $\mathrm{WL}_{\mathrm{SG}}$ for the multipolar sample and for the combined sample are displayed in Figure 5. The left panel of Figure 5 is the correlation plot of $\mathrm{WL}_{\mathrm{SS}}$ and $\mathrm{WL}_{\mathrm{SG}}$ for our sample of multipolar active regions. Of these 16 multipolar active regions, $8(50 \%)$ produced CMEs in the $0-72 \mathrm{hr}$ window (Fig, 5, left), whereas of the 40 active regions in our bipolar sample, only $11(28 \%)$ produced CMEs in this window (Fig. 4, right). This demonstrates that multipolar active regions are strikingly more CME productive than are purely bipolar and predominantly bipolar active regions.

For our multipolar sample, $\mathrm{WL}_{\mathrm{SS}}$ and $\mathrm{WL}_{\mathrm{SG}}$ both have the same CME-prediction success rate, $75 \% \pm 11 \%$. This is not significantly different than the CME-prediction success rates for the bipolar sample: $80 \% \pm 6 \%$ for $\mathrm{WL}_{\mathrm{SS}}$ and $75 \% \pm 7 \%$ for $\mathrm{WL}_{\mathrm{SG}}$ (Fig. 4, right). The statistical uncertainties in the CME-prediction success rates are almost 2 times larger for the multipolar sample (Fig. 5, left) than for the bipolar sample (Fig. 4, right) because the multipolar sample size (16) is 2.5 times smaller than the bipolar sample size (40). For the combined sample (Fig. 5, right), $\mathrm{WL}_{\mathrm{SS}}$ and $\mathrm{WL}_{\mathrm{SG}}$ again turn out to have equal CME-prediction success rates, $79 \% \pm 5 \%$, which again is not significantly different than those from the bipolar sample (Fig. 4, right). Similarly, 

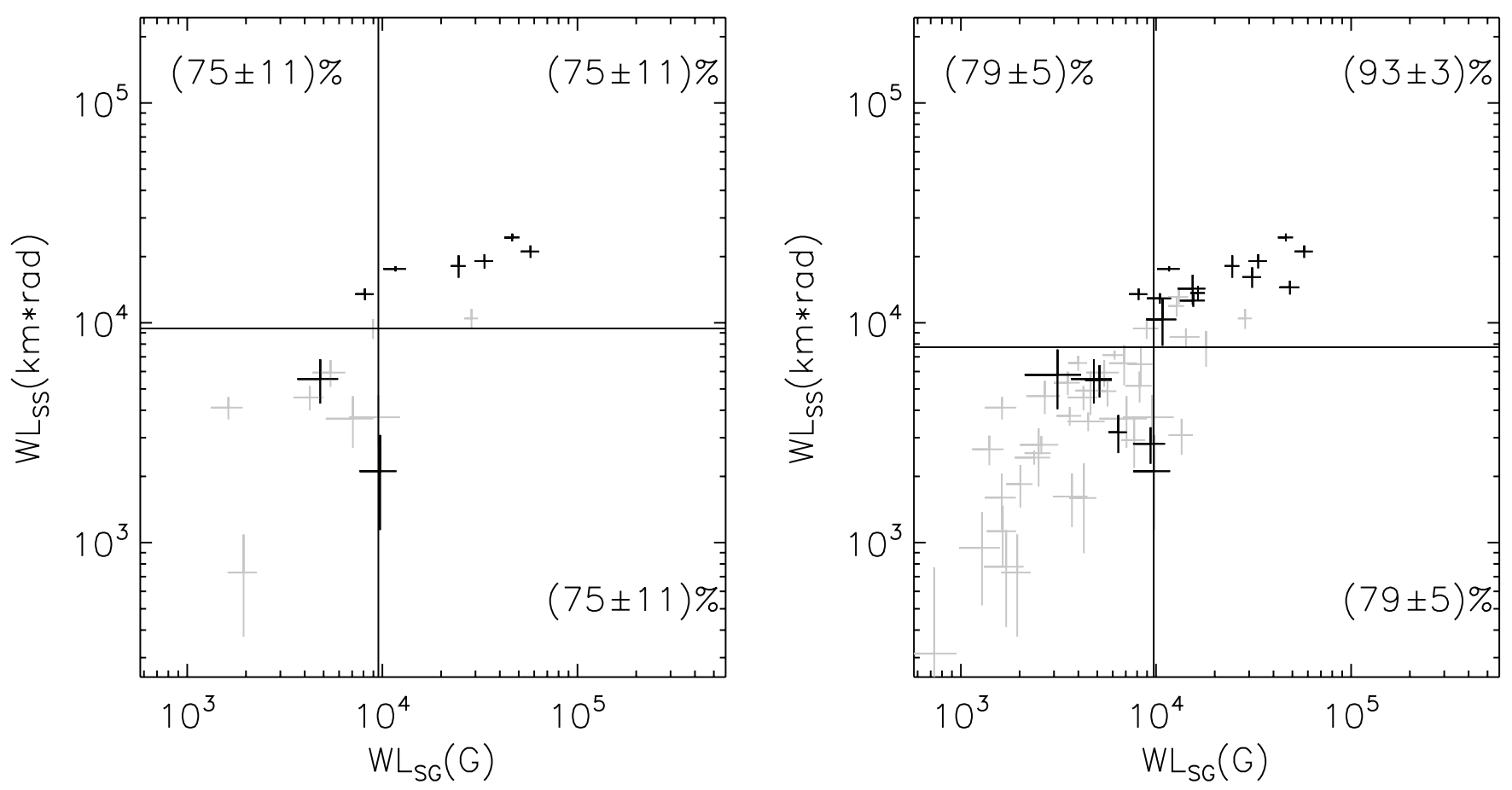

FIG. 5.-Correlation plots showing that for prediction of CME production the two weighted all-neutral-line measures of total nonpotentiality work equally well for active regions of any degree of magnetic complexity. Left: Correlation plot of $\mathrm{WL}_{\mathrm{SG}}$ and $\mathrm{WL}_{\mathrm{SS}}$ for our sample of 16 multipolar active regions. Right: Correlation plot of $\mathrm{WL}_{\mathrm{SG}}$ and $\mathrm{WL}_{\mathrm{SS}}$ for the combined sample of 56 active regions, 40 bipolar and 16 multipolar. These plots have the same format at those in Figs. 3 and 4 , and are again for the $0-72 \mathrm{hr}$ forecast window. To within the statistical uncertainty, the CME-prediction success rates of $\mathrm{WL}_{\mathrm{SG}}$ and $\mathrm{WL}_{\mathrm{SS}}$ found here for either of these two samples are equal to those found in the right panel of Fig. 4 for the bipolar sample alone.

the agreement success rate of $\mathrm{WL}_{\mathrm{SS}}$ and $\mathrm{WL}_{\mathrm{SG}}$ for the combined sample, $93 \% \pm 3 \%$, is the same as for the bipolar sample, $95 \% \pm$ $3 \%$, to within the statistical uncertainty. Overall, from the results given in Figures 3, 4 and 5, we conclude that for our method of quantitatively assessing the magnetic preconditions for activeregion CME production, $\mathrm{WL}_{\mathrm{SS}}$ and $\mathrm{WL}_{\mathrm{SG}}$ are magnetogram measures of active-region total nonpotentiality that perform as well for active regions of any degree of magnetic complexity as the measures $L_{\mathrm{SSM}}$ and $L_{\mathrm{SGM}}$ perform for purely and predominantly bipolar active regions.

\subsection{Performance of the Line-of-Sight Magnetogram Measure of Active-Region Total Nonpotentiality}

Next, we come to the performance of ${ }^{L} \mathrm{WL}_{\mathrm{SG}}$, our weighted all-neutral-line measure of active-region total nonpotentiality that is measured from a line-of-sight magnetogram in the same way that $\mathrm{WL}_{\mathrm{SG}}$ is measured from the vertical-field component of a vector magnetogram. For active regions close enough to disk center, the line-of-sight magnetogram approximates the verticalfield magnetogram well enough that ${ }^{L} \mathrm{WL}_{\mathrm{SG}}$ is practically equal to $\mathrm{WL}_{\mathrm{SG}}$ and so is a good proxy for $\mathrm{WL}_{\mathrm{SG}}$. As we will now show, it turns out that active regions that are within $30^{\circ}$ of disk center are close enough to disk center for ${ }^{L} \mathrm{WL}_{\mathrm{SG}}$ to be a good approximation for $\mathrm{WL}_{\mathrm{SG}}$.

Figure 6 is the correlation plot of $\mathrm{WL}_{\mathrm{SG}}$ and ${ }^{L} \mathrm{WL}_{\mathrm{SG}}$ for our sample of 41 vector magnetograms of active regions within $30^{\circ}$ of disk center and for the $0-72 \mathrm{hr}$ window. This sample includes active regions of all degrees of magnetic complexity: purely bipolar, predominantly bipolar, and multipolar. In Figure $6,{ }^{L} \mathrm{WL}_{\mathrm{SG}}$ is obviously well correlated with $\mathrm{WL}_{\mathrm{SG}}$ throughout the range of total nonpotentiality, even though on average the value of ${ }^{L} \mathrm{WL} \mathrm{L}_{\mathrm{SG}}$ is only about half that of $\mathrm{WL}_{\mathrm{SG}}$ for the same active region. For this sample, the agreement success rate between $\mathrm{WL}_{\mathrm{SG}}$ and ${ }^{L} \mathrm{WL}$

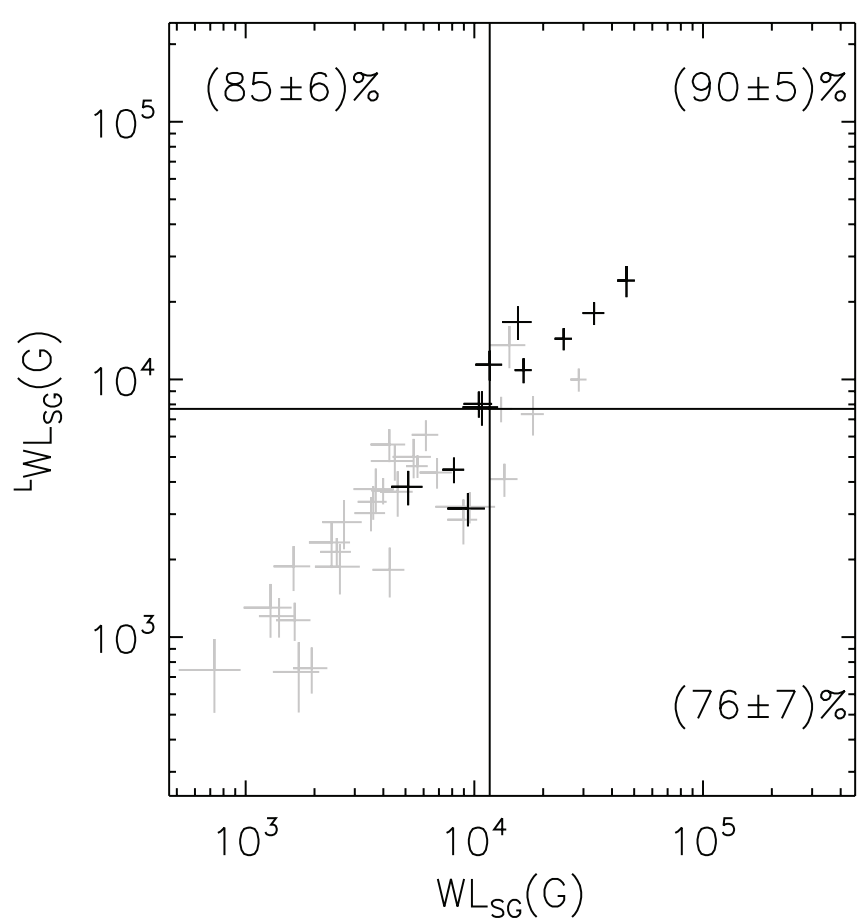

FIG. 6.-Correlation plot of the weighted all-neutral-line magnetic-gradient measure of total nonpotentiality, $\mathrm{WL}_{\mathrm{SG}}$, and this measure's proxy, ${ }^{\mathrm{L}} \mathrm{WL} \mathrm{L}_{\mathrm{SG}}$, showing that for active regions within $30^{\circ}$ of disk center ${ }^{L} \mathrm{WL}_{\mathrm{SG}}$ works as well as $\mathrm{WL}_{\mathrm{SG}}$ for CME prediction. The plot is for a subset of 41 active regions from our combined bipolar-plus-multipolar sample of 56 active regions, the 41 that were within $30^{\circ}$ of disk center at the times of their measured magnetograms. For this sample, to within the statistical uncertainty, the CME-prediction success rate of ${ }^{L} \mathrm{WL}_{\mathrm{SG}}$, $85 \% \pm 6 \%$, equals that of $\mathrm{WL}_{\mathrm{SG}}, 76 \% \pm 7 \%$. The plot has the same format as the other correlation plots and is for the same forecast window, $0-72 \mathrm{hr}$. 
is $90 \% \pm 5 \%$, which, within the statistical uncertainty, is the same as the $93 \% \pm 3 \%$ agreement success rate between $\mathrm{WL}_{\mathrm{SS}}$ and $\mathrm{WL}_{\mathrm{SG}}$ for the combined sample (Fig. 5, right). Moreover, in Figure 6 , the CME-prediction success rate of ${ }^{L} \mathrm{WL}_{\mathrm{SG}}$ is $85 \% \pm 6 \%$, which equals the $76 \% \pm 7 \%$ CME-prediction success rate of $\mathrm{WL}_{\mathrm{SG}}$ to within the statistical uncertainty. We conclude from the results in Figure 6 that for active regions within $30^{\circ}$ of disk center ${ }^{L} \mathrm{WL}_{\mathrm{SG}}$ is an adequately good all-neutral-line measure of activeregion total nonpotentiality that can be measured from a line-ofsight magnetogram instead of a vector magnetogram.

\subsection{Variation of CME-Prediction Success Rate with Length of Forecast Window}

In Figures 3, 4, 5, and 6, for each of our neutral-line measures of active-region total nonpotentiality, we have presented the CME-prediction success rate for the $0-72 \mathrm{hr}$ forecast window. In each case, we also found the CME-prediction success rate for each of four other forecast windows: $0-24 \mathrm{hr}, 0-48 \mathrm{hr}, 0-96 \mathrm{hr}$, and $0-120 \mathrm{hr}$. For each combination of measure and sample, the CME-prediction success rate for each of the five windows is given in Table 1. For each measure, with respect to the statistical uncertainties in the CME-prediction success rates, there is little significant difference in the success rates for the other windows from that for the $0-72 \mathrm{hr}$ window, except for the $0-120 \mathrm{hr}$ window, which often has a significantly smaller success rate. In most cases, the success rate is somewhat smaller for the 0-96 hr window than for the $0-72 \mathrm{hr}$ window, but not significantly smaller. This behavior may have the following plausible explanation. We assume that the total nonpotentiality of most active regions evolves gradually, taking a day or so to increase or decrease by a factor of 2 or so. For the first 3 days after the magnetogram, the degradation in the prediction resulting from the change in total nonpotentiality is offset by the increase in the chance of a CME occurring for progressively longer time windows, for active regions that are initially sufficiently nonpotential. After 3 days, the cumulative evolution of the total nonpotentiality overpowers this effect, and the CME-prediction success rate begins to drop.

\section{SUMMARY AND DISCUSSION}

This paper is a further development of our previous work, reported most recently in Falconer et al. (2006), in which we have investigated the magnetic causes of CMEs by means of measures of active-region nonpotentiality that can be measured from active-region magnetograms and the performance of these measures as predictors of active-region CME productivity. Our previous investigations were restricted to nominally bipolar active regions, which are purely bipolar or predominantly bipolar active regions having a clearly identifiable main neutral line, because the measures of active-region nonpotentiality that we had devised were well defined only for nominally bipolar active regions, not for magnetically more complex multipolar active regions. In particular, two of the magnetogram measures of active-region total nonpotentiality used in our previous work, $L_{\mathrm{SSM}}$ and $L_{\mathrm{SGM}}$, by definition, are measured from along the main neutral line in a vector magnetogram of the active region, and hence are undefined for complex active regions that have no clear main neutral line.

It is well known that multipolar active regions produce more flares and CMEs than do nominally bipolar active regions (e.g., Svestka 1976; Zirin 1988). For this reason, in the present paper, we have introduced two new measures of active-region total nonpotentiality, $\mathrm{WL}_{\mathrm{SS}}$ and $\mathrm{WL}_{\mathrm{SG}}$. These are generalized versions of $L_{\mathrm{SSM}}$ and $L_{\mathrm{SGM}}$ that are defined for active regions of any degree of magnetic complexity, nominally bipolar or multipolar, and that, for active regions of low to moderate total nonpotentiality, are measured with greater precision than are $L_{\mathrm{SSM}}$ and $L_{\mathrm{SGM}}$. The measure $\mathrm{WL}_{\mathrm{SS}}$ is the integral of the shear angle of the horizontal component of the magnetic field over all strong-field neutral lines in the vector magnetogram, and the measure $\mathrm{WL}_{\mathrm{SG}}$ is the integral of the horizontal gradient of the vertical component of the magnetic field over all strong-field neutral lines in the vector magnetogram.

By testing $\mathrm{WL}_{\mathrm{SS}}$ and $\mathrm{WL}_{\mathrm{SG}}$ on a sample of 40 nominally bipolar active regions and 16 multipolar active regions we found the following.

1. For nominally bipolar active regions, as predictors of an active region's CME productivity in the next few days after the magnetogram, $\mathrm{WL}_{\mathrm{SS}}$ and $\mathrm{WL}_{\mathrm{SG}}$ perform equally well, and each performs as well as either $L_{\mathrm{SSM}}$ or $L_{\mathrm{SGM}}$.

2. For nominally bipolar active regions of low to moderate total nonpotentiality, the uncertainties in the measurements of $\mathrm{WL}_{\mathrm{SS}}$ and $\mathrm{WL}_{\mathrm{SG}}$ are much less than in the measurements of $L_{\mathrm{SSM}}$ and $L_{\mathrm{SGM}}$. This results in the correlation between $\mathrm{WL}_{\mathrm{SS}}$ and $\mathrm{WL}_{\mathrm{SG}}$ being much tighter than that between $L_{\mathrm{SSM}}$ and $L_{\mathrm{SGM}}$ for active regions of low to moderate total nonpotentiality.

3. As predictors of active-region CME productivity, $\mathrm{WL}_{\mathrm{SS}}$ and $\mathrm{WL}_{\mathrm{SG}}$ both perform as well for multipolar active regions as for nominally bipolar active regions.

4. The CME-prediction success rate for either $\mathrm{WL}_{\mathrm{SS}}$ or $\mathrm{WL}_{\mathrm{SG}}$ is about $75 \%$ for forecast windows from $0-24 \mathrm{hr}$ to $0-$ $96 \mathrm{hr}$, and drops to about $65 \%$ for the $0-120 \mathrm{hr}$ window. This result is plausibly due to the time for a large evolutionary change in an active region's total nonpotentiality being typically several days.

In light of the above results, $\mathrm{WL}_{\mathrm{SS}}$ and $\mathrm{WL}_{\mathrm{SG}}$ are the allneutral-line measures of active-region total nonpotentiality that we plan to use from now on in developing methods of forecasting active-region CME productivity from vector magnetograms.

In addition, we showed that ${ }^{L} \mathrm{WL}_{\mathrm{SG}}$, the measure of activeregion total nonpotentiality that corresponds to $\mathrm{WL}_{\mathrm{SG}}$ but is measured from a line-of-sight magnetogram instead of from the vertical-field component of a vector magnetogram, performs as well as $\mathrm{WL}_{\mathrm{SG}}$ as a CME predictor for active regions within 30 heliographic degrees of disk center. This opens the full-disk lineof-sight magnetograms from $\mathrm{SOHO}$ MDI to our analysis. The 96 minute cadence and nearly continuous coverage of this data set since the advent of $\mathrm{SOHO}$ in 1996 will allow us to measure the total nonpotentiality and its evolution for hundreds of active regions over the $3-4$ days that they were within $30^{\circ}$ of disk center. We will study this evolution (1) qualitatively for patterns that reflect the magnetic conditions or processes that yield CMEs, and (2) quantitatively for its importance as a predictor of activeregion $\mathrm{CME}$ productivity.

We have purposely limited the present paper to results from vector magnetograms from one magnetograph to establish the following

1. That we can apply these measures to active regions of any degree of magnetic complexity to gauge the nonpotentiality of an active region.

2 . That the nonpotentiality of active regions of any degree of magnetic complexity is strongly correlated with CME productivity.

3. That one of our measures of total nonpotentiality can be adequately measured from line-of-sight magnetograms for active regions within $30^{\circ}$ of disk center. To demonstrate this, we evaluated ${ }^{L} \mathrm{WL}_{\mathrm{SG}}$ from only the line-of-sight component of the MSFC vector magnetograms. 
We have measured $\Phi$ and ${ }^{L} \mathrm{WL}_{\mathrm{SG}}$ from MDI line-of-sight magnetograms for an initial sample of 46 active regions, and we are now writing a paper on the results for submission to The Astrophysical Journal.

This work was supported by funding from NSF's Division of Atmospheric Sciences through its SHINE and Space Weather programs, and by NASA's Science Mission Directorate through the Living With a Star Targeted Research and Technology program of its Heliophysics Division. We acknowledge the use of the SOHO LASCO CME Catalog generated and maintained by the Center of Solar Physics and Space Weather of the Catholic University of America in cooperation with the Naval Research Laboratory and NASA. SOHO is a project of international cooperation between ESA and NASA.
Canfield, R. C., Hudson, H. S., \& Mckenzie, D. E. 1999, Geophys. Res. Lett., 26, 627

Falconer, D. A. 2001, J. Geophys. Res., 106, 25185

Falconer, D. A., Moore, R. L., \& Gary, G. A. 2002, ApJ, 569, 1016 . 2003, J. Geophys. Res., 108, 1380 2006, ApJ, 644, 1258

Falconer, D. A., Moore, R. L., Porter, J. G., Gary, G. A., \& Shimzu, T. 1997, ApJ, 482, 519

Gary, G. A., Moore, R. L., Hagyard, M. J., \& Haisch, B. M. 1987, ApJ, 314, 782

Gosling, J. T. 1996, ARA\&A, 34, 35

Kahler, S. 1987, Rev. Geophys., 25, 663

Krimigis, S. M. 1992, in The Astronomy and Astrophysics Encyclopedia, ed. S. P. Maran (New York: Van Nostrand Reinhold), 332

Lapin, L. 1978, Statistics for Modern Business Decisions (New York: Harcourt Brace Jovanovich)

Martin, S. F. 1998, Sol. Phys., 182, 107

Moore, R. L. 1992, in The Astronomy and Astrophysics Encyclopedia, ed. S. P. Maran (New York: Van Nostrand Reinhold), 637

\section{REFERENCES}

Moore, R. L., Sterling, A. C., Hudson, H. S., \& Lemen, J. R. 2001, ApJ, 552, 833

Reames, D. V. 1999, Space Sci. Rev., 90, 413

2001, Space Weather, ed. P. Song, H. J. Singer, \& G. L. Siscoe (Geophys. Monogr. 125; Washington: AGU)

Sturrock, P. A. 1980, Solar Flares (Boulder: Colorado Assoc. Univ. Press)

Suess, S. T., \& Tsurutani, B. T. 1998, From the Sun: Auroras, Magnetic Storms, Solar Flares, Cosmic Rays (Washington: AGU)

Svestka, Z. 1976, Solar Flares (Dordrecht: Reidel)

Svestka, Z., Jackson, B. V., \& Machado, M. E. 1992, Eruptive Solar Flares (Berlin: Springer)

Wagner, W. J. 1984, ARA\&A, 22, 267

Wang, Y., \& Zhang, J. 2007, ApJ, 665, 1428

Webb, D. F., Krieger, A. S., \& Rust, D. M. 1976, Sol. Phys., 48, 159

Yashiro, S., Gopalswamy, N., Michalek, G., St. Cyr, O. C., Plunkett, S. R., Rich, N. B., \& Howard, R. A. 2004, J. Geophys. Res., 109, A07105 Zirin, H. 1988, Astrophysics of the Sun (Cambridge: Cambridge Univ. Press) Zirin, H., \& Liggett, M. A. 1987, Sol. Phys., 113, 267 